\title{
AVALIAÇÃO DO DESEMPENHO DE UM SISTEMA DE TRATAMENTO UTILIZANDO OS PROCESSOS ELETROCOAGULAÇÃO E FOTO-FENTON INTEGRADOS NO TRATAMENTO DE UM EFLUENTE TÊXTIL
}

\author{
Diego Ricieri Manenti, ${ }^{1,2,3}$ \\ Fernando Henrique Borba ${ }^{1}$ \\ Aparecido Nivaldo Módenes ${ }^{2}$ \\ Fernando Rodolfo Espinoza-Quiñones ${ }^{2}$ \\ Soraya Moreno Palácio ${ }^{2}$ \\ Vitor Jorge Pais Vilar ${ }^{3}$ \\ Rosângela Bergamasco ${ }^{1}$
}

\begin{abstract}
Resumo: O principal objetivo deste trabalho foi avaliar a eficiência de um sistema de tratamento de efluentes têxteis composto pelos processos eletrocoagulação (EC) e fotoFenton solar (FFS) integrados. Utilizando reatores de escala laboratorial, do tipo sequencial, o efluente têxtil foi tratado pela EC seguido pelo processo FFS. Os parâmetros operacionais do reator FFS foram otimizados realizando um delineamento experimental $3^{3}$ completo, seguido da análise estatística dos dados obtidos. O efluente têxtil bruto apresentou uma coloração preta, turbidez moderada, alta alcalinidade e elevada carga orgânica (DQO $=1636 \mathrm{mg} \mathrm{O}_{2} \mathrm{~L}^{-1}$, COT $=638 \mathrm{mg} \mathrm{C} \mathrm{L}{ }^{-1}$ ). Em $20 \mathrm{~min}$ os processos integrados EC/FFS apresentaram reduções de 99,5\% da DQO, 96\% do COT, 99,9\% da cor e da turbidez, atendendo os valores máximos permitidos pela legislação brasileira para despejo de efluentes em corpos hídricos receptores. Assim, a integração dos processos EC/FFS mostrou-se uma alternativa eficaz no tratamento de efluente têxtil.
\end{abstract}

Palavras-chave: efluente têxtil; integração de processos; eletrocoagulação; foto-Fenton.

Abstract: This paper aimed to evaluate the efficacy of an integrated textile wastewater treatment system based on both electrocoagulation and solar photo-Fenton processes. A sequential-type, lab-scale reactors system was built and used. By performing a complete experimental design $3^{3}$, analyzing second order models for some response variables such as Chemical Oxygen Demand, Turbidity and color as well as an ANOVA evaluation, the set of operating photo-Fenton reactor parameters was optimized. High contents of organic matter, intense dark color, moderate turbidity and high alkalinity are characteristics of textile wastewater samples. High performance in COD, DOC, color and turbidity removal was attended by the integrated textile wastewaters treatment system operated in 20 min and set at optimized operating reactors parameters, with their final concentrations below the maximum allowed limits recommended by the Brazilian environmental legislation. Thus, an integration of electrocoagulation and photo-Fenton processes is an alternative effective method on removals of main pollutants of textile effluents.

Keywords: textile wastewater; integrated processes; electrocoagulation; photo-Fenton.

\footnotetext{
${ }^{1}$ Programa de Pós-Graduação em Engenharia Química da Universidade Estadual de Maringá.

${ }^{2}$ Programa de Pós-Graduação em Engenharia Química da Universidade Estadual do Oeste do Paraná.

${ }^{3}$ LSRE - Laboratory of Separation and Reaction Engineering - Associated Laboratory LSRE/LCM,

Faculdade de Engenharia, Universidade do Porto, Portugal.
} 


\section{INTRODUÇÃO}

Processos industriais como o do setor têxtil, requerem elevados volumes de água que são convertidos em efluentes com características complexas contendo poluentes orgânicos e inorgânicos que são carcinogênicos e mutagênicos aos humanos e tóxicos para as vidas aquáticas (Lucas e Peres, 2006). O despejo destes efluentes em corpos hídricos depende da eficiência dos sistemas de tratamento em atingir os valores limites de emissão dos parâmetros legais de controle ambiental impostos pelos órgãos competentes.

Os sistemas de tratamento, geralmente compostos por processos físicos, físico-químicos, químicos $\mathrm{e}$ biológicos nem sempre são eficientes na redução destes compostos (Yeber et al., 1998; Wang e Kutal, 2001). Por esta razão, tem sido proposto tecnologias alternativas e sistemas de tratamento integrados. A integração de processos consiste em adotar tratamentos prévios ou posteriores, de naturezas distintas, visando em geral o aumento da eficiência global do sistema, à redução da área de instalação e dos custos, ou à transformação química dos poluentes em substâncias biologicamente degradáveis ou em substâncias mais facilmente elimináveis por processos físico-químicos convencionais.

Os processos químicos podem ser empregados isoladamente ou em combinação entre eles com um tratamento prévio ou polimento final. A integração mais investigada nos últimos anos é a de técnicas emergentes, como eletroquímicas e oxidação avançada com processos biológicos (Vilar et al., 2011a; Rocha et al., 2011; Pintor et al., 2011; Rosales et al., 2012; Módenes et al., 2012a).

Os processos oxidativos avançados (POA) envolvem agentes de espécies transitórias oxidantes, principalmente os radicais hidroxila, que transformam a grande maioria dos poluentes orgânicos em dióxido de carbono, água e ânions inorgânicos. Dentre os POA, os mais utilizados são o Fenton e o foto-Fenton (Babuponnusami e Muthukumar, 2012;
Monteagudo et al., 2010). O processo foto-Fenton tem sido aplicado na redução de corantes (Manenti et al., 2010; Katsumata et al., 2010; Devi et al., 2011; Prato-Garcia e Buitrón, 2012), trihalometanos (Moncayo-Lasso et al., 2012), antibióticos (Pérez-Moya et al., 2010) e no tratamento de efluentes industriais como: lixiviados (Rocha et al., 2011; Vilar et al., 2011a), cozedura de cortiça (Pintor et al., 2011), processamento de alimentos (Manenti et al., 2009), vinícolas, curtume (Monteagudo et al., 2012; Borba et al., 2013) e têxteis (Vilar et al., 2011b; Módenes et al., 2012b).

Outra técnica que tem sido investigada no tratamento de resíduos líquidos industriais é a eletrocoagulação (EC). Beneficiada de oxidações eletroquímicas, a EC tem apresentado elevada eficiência na remoção de poluentes orgânicos e inorgânicos de efluentes (Mollah et al., 2001), tais como: têxteis (Palácio et al., 2009; Merzouk et al., 2011; Yilmaz et al., 2011), galvanoplastia (Espinoza-Quiñones et al., 2012), curtume (Espinoza-Quiñones et al., 2009; Módenes et al., 2012a), processamento de alimentos (Borba et al., 2010), fármacos (Farhadi et al., 2012) e metais pesados (Al Aji et al., 2012).

Desta forma, o principal objetivo deste trabalho foi avaliar a eficiência de um sistema de tratamento de efluentes têxteis composto pelos processos EC e FFS integrados. Utilizando reatores de escala laboratorial, do tipo sequencial, o efluente foi pré-tratado pela EC seguido pelo processo FFS. Os parâmetros operacionais da EC foram baseados em resultados prévios (Palácio et al., 2009), enquanto que $o$ processo FFS foi otimizado aplicando um delineamento experimental $3^{3}$ completo, cujos dados foram avaliados estatisticamente utilizando a metodologia das superfícies de respostas. A eficiência dos processos EC, FFS e EC/FFS foi avaliada baseada na redução dos parâmetros físicoquímicos DQO, COT, cor e turbidez. 


\section{MATERIAIS E MÉTODOS}

\subsection{Amostras e reagentes}

O efluente têxtil utilizado neste trabalho consiste de águas residuais homogeneizadas, coletadas após os processos de tingimento e lavagem de tecidos de uma planta industrial localizada na cidade de Toledo/PR. Após as coletas, as amostras foram conservadas de acordo com a metodologia descrita no Standard Methods (APHA, 2005).

$\mathrm{Na}$ realização dos experimentos do sistema integrado eletrocoagulação/fotoFenton solar (EC/FFS) foram utilizados peróxido de hidrogênio $30 \% \quad(\mathrm{v} / \mathrm{v})$ (Sinth), sulfato ferroso heptahidratado (Merck), enquanto que hidróxido de sódio (Merck) e ácido sulfúrico concentrado (Synth) foram utilizados nos ajustes do $\mathrm{pH}$, quando requerido. Outros produtos químicos, reagentes e solventes utilizados apresentam grau de pureza analítico sem purificação prévia.

\subsection{Reatores de eletrocoagulação e foto-Fenton solar}

Os experimentos de EC foram realizados utilizando as condições ótimas operacionais, intensidade de corrente de 5 A e o pH inicial da solução em 7,0, conforme resultados obtidos previamente (Palácio et al., 2009). Baseado nestas informações foi utilizado um reator de
EC de escala laboratorial (ver Figura 1), constituído por um bécker de borossilicato de 1,5 L (14,4 $\mathrm{cm}$ de altura e 11,6 cm de diâmetro), uma placa de agitação magnética (ARE UNI-3650) para homogeneizar a solução, e um conjunto de eletrodos de ferro, monopolar, acoplado em paralelo a uma fonte digital estabilizadora de corrente contínua $(0-10$ A) e voltagem $(0-30 \mathrm{~V})$ (Instrutemp DC Power Supply FA 1030). O conjunto de eletrodos é constituído por oito placas $(10 \mathrm{~cm}$ de altura x $5 \mathrm{~cm}$ de largura $\times 0,15 \mathrm{~cm}$ de espessura) de ferro, separadas a uma distância de $0,5 \mathrm{~cm}$ entre ambas. Os eletrodos foram parcialmente imersos na solução, com área superficial efetiva de $350 \mathrm{~cm}^{2}$.

Os experimentos FFS foram realizados utilizando as condições ótimas operacionais, $0,5 \mathrm{~g} \mathrm{Fe}^{2+} \mathrm{L}^{-1}, 6 \mathrm{~g} \mathrm{H}_{2} \mathrm{O}_{2} \mathrm{~L}^{-1}$ e pH 3,0, conforme resultados prévios (Módenes et al., 2012b). Baseado nestas informações, o fotoreator consiste em um bécker de borossilicato de $200 \mathrm{~mL}(9,3$ $\mathrm{cm}$ de altura e 7,8 $\mathrm{cm}$ de diâmetro), e um agitador magnético (ARE UNI-3650) para homogeneizar a solução (Figura 2). $\mathrm{O}$ reator foi montado em um ambiente externo durante o verão (Dez.-2010 a Fev.-2011), no período das 11 e $14 \mathrm{~h}$, utilizando a luz solar como fonte de radiação UV. Segundo o Instituto Nacional de Pesquisas Espaciais (INPE),

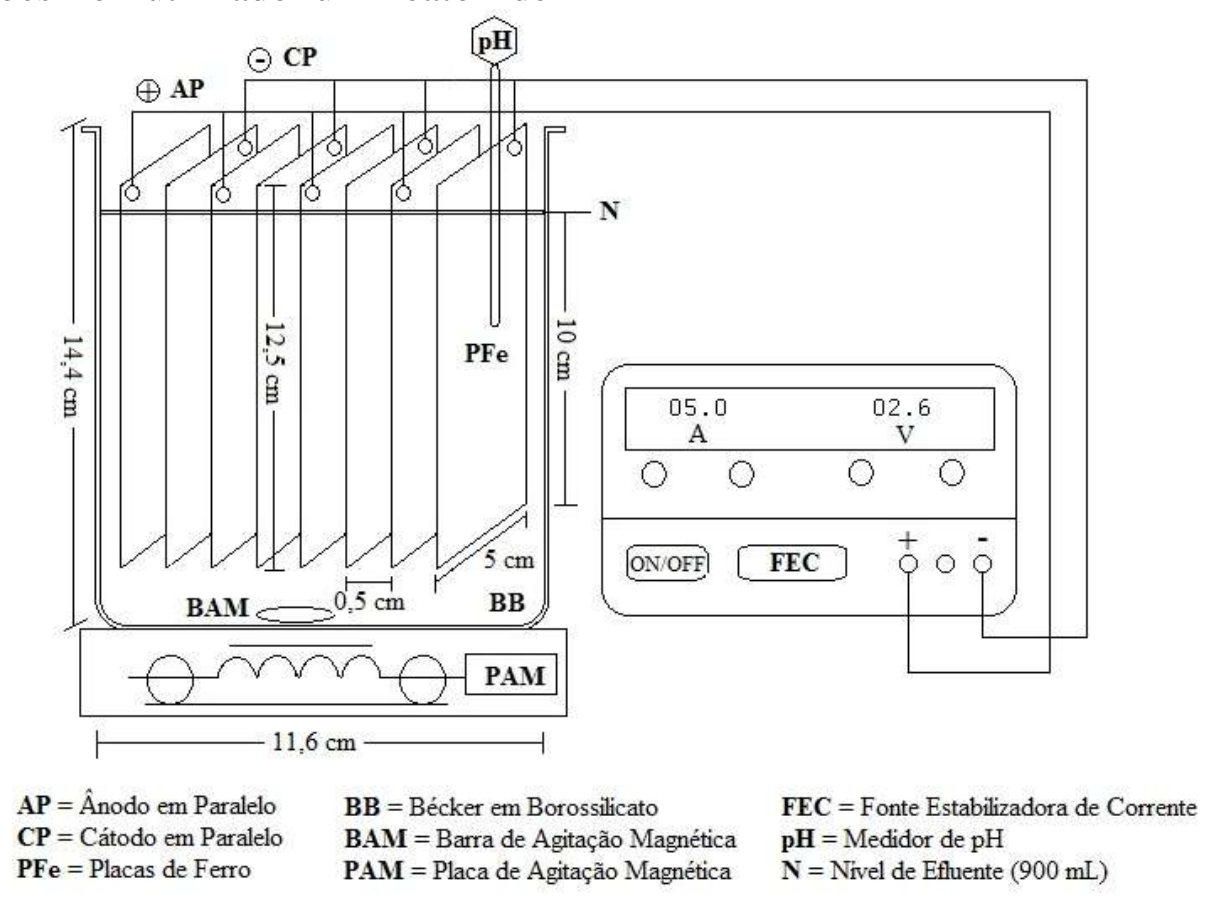

Figura 1. Esquema da instalação do EC-reator de escala laboratorial. 


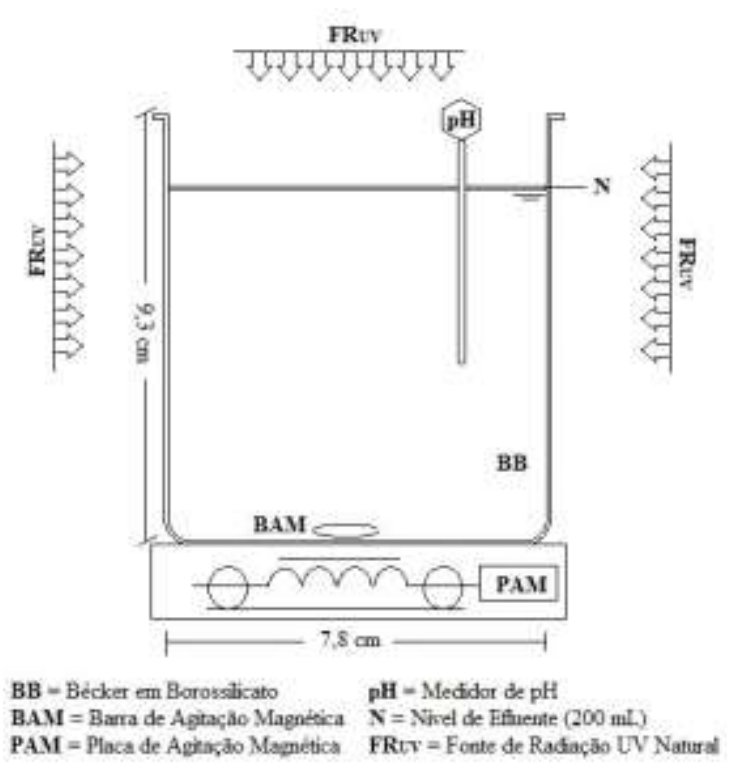

Figura 2. Esquema da instalação do fotoreator de escala laboratorial.

a intensidade média integrada da irradiação solar, neste período, na região de Toledo-PR (latitude 2441'07" S, longitude 53०41'48" W) foi de 13,6 MJ $\mathrm{m}^{-2}$. Parte desta radiação está no espectro UV utilizado na faixa de trabalho da reação FFS.

\subsection{Procedimentos experimentais}

Para os experimentos da EC foram utilizados $900 \mathrm{~mL}$ do efluente têxtil com o pH ajustado para 7,0. O conjunto de eletrodos foi imerso na solução constantemente agitada $(\approx 900 \mathrm{rpm})$. Após, a fonte de alimentação foi ligada mantendo a intensidade de corrente contínua em 5 A e a voltagem em cerca de 2,7-3,6 V. Para evitar perdas de eficiência devido a passivação das placas de ferro, conforme descrito em EspinozaQuiñones et al. (2009), foram realizadas inversões de polaridade dos eletrodos a cada $30 \mathrm{~min}$ de eletrólise. Após cada ensaio, o efluente permaneceu em repouso $(\approx 30 \mathrm{~min})$ para separar os materiais flutuantes, o precipitado e as amostras tratadas.

Para os experimentos FFS foram utilizados $200 \mathrm{~mL}$ de efluente constantemente agitados $(\approx 900 \mathrm{rpm})$ no interior do reator. Antes de cada ensaio, o $\mathrm{pH}$ foi ajustado para os valores prédeterminados. Após, foram adicionadas em ordem, as alíquotas das soluções de ferro e $\mathrm{H}_{2} \mathrm{O}_{2}$ nas concentrações pré- determinadas, e a mistura reacional foi exposta a radiação solar.

Baseado nos resultados ótimos da EC e do FFS publicados em trabalhos prévios (Palácio et al., 2009; Módenes et al., 2012b), foram realizados testes preliminares com os reatores operando em combinado. As melhores respostas foram obtidas com o pré-tratamento via EC seguido pelo processo FFS. Sendo que acima de 5 min de eletrólise não ocorrem variações significativas na eficiência global do tratamento. Desta forma, foi realizado o tratamento do efluente bruto durante $5 \mathrm{~min}$ de EC seguido do processo FFS.

Após o processo EC, o efluente foi submetido ao processo FFS. Foram otimizados os parâmetros operacionais do reator (POR) (concentração de $\mathrm{H}_{2} \mathrm{O}_{2}, \mathrm{pH}$ inicial e tempo de irradiação UV) através de experimentos delineados conforme o Planejamento Experimental $3^{3}$ Completo (PEC). Os níveis dos parâmetros operacionais estudados são apresentados na Tabela 1. Nesta etapa não foi realizado um estudo da influência da concentração de ferro, pois durante a EC ocorre um desgaste das placas de ferro do conjunto de eletrodos ocasionando em uma elevação da concentração deste em níveis satisfatórios para a realização do processo FFS (cerca de $34 \mathrm{mg} \mathrm{Fe}^{2+} \mathrm{L}^{-1}$ ).

Tabela 1. Níveis dos POR estudados após a EC.

\begin{tabular}{lcccc}
\hline \multirow{2}{*}{ POR } & \multirow{2}{*}{ Variáveis } & \multicolumn{3}{c}{ Níveis } \\
& & -1 & 0 & 1 \\
\hline$\left[\mathrm{H}_{2} \mathrm{O}_{2}\right]\left(\mathrm{mg} \mathrm{L}^{-1}\right)$ & $q_{1}$ & 1 & 4 & 7 \\
pH inicial & $q_{2}$ & 3 & 6 & 9 \\
Tempo (min.) & $q_{3}$ & 60 & 120 & 180 \\
\hline
\end{tabular}

Para otimizar o sistema integrado EC/FFS, foi realizada uma abordagem estatística aplicada nos dados do PEC, baseada na Metodologia das Superfícies de Respostas (MSR) (Meyers e Montgomery, 2002; Khuri e Mukhopadhyay, 2010) a partir de um modelo polinomial de $2^{\mathrm{a}}$ ordem conforme apresentado na Equação 1.

$$
\begin{aligned}
& R=a_{0}+\sum_{i=1}^{3} a_{i} q_{j}+\sum_{i=1}^{3} \sum_{j=1}^{3} b_{i j} q_{i} q_{j} \\
& +\sum_{i=1}^{3} \sum_{j=1}^{3} \sum_{k=1}^{3} w_{i j k} q_{i} q_{j} q_{k}+\sum_{i=1}^{3} \sum_{j=1}^{3} v_{i j} q_{i}^{2} q_{j}^{2}
\end{aligned}
$$


Os resultados experimentais foram analisados através do pacote estatístico Statistica (Copyright 1984-2000 by statsoft, Inc) no modo "experimental design", para três níveis de variação e três modos de interação, gerando os valores dos coeficientes do modelo $\left(a_{0}\right)$, lineares (a), quadráticos e suas interações $(b, w$ e v). Para validar a reprodução dos dados pelo modelo proposto, foi realizada uma análise de variância (ANOVA) com um nível de confiança de 95\% ( $p<0,05)$. Uma representação gráfica 3-D de todas as respostas estatísticas foi obtida através da aplicação dos critérios de Lagrange (Ferreira et al., 2004).

Com os reatores EC, FFS e EC/FFS operando nas condições ótimas, foram retiradas alíquotas após 5, 10, 15, 20, 30, 45, 60, 90 e $120 \mathrm{~min}$, para verificar a eficiência de cada processo na redução dos compostos orgânicos (DQO, COT, cor e turbidez) do efluente têxtil.

\subsection{Determinações analíticas}

A concentração de $\mathrm{H}_{2} \mathrm{O}_{2}$ foi determinada utilizando o método do metavanadato de amônia (Nogueira et al., 2005). Este método é baseado na reação do $\mathrm{H}_{2} \mathrm{O}_{2}$ com o metavanadato em meio ácido, formando o cátion peroxovanádio de cor vermelho-alaranjado que apresenta absorvância máxima em $450 \mathrm{~nm}$. A concentração de ferro total dissolvido foi determinada através do método colorimétrico com 1,10-fenantrolina de acordo com a norma ISO 6332 (1988). A demanda química de oxigênio (DQO) foi determinada pelo método colorimétrico de refluxo fechado conforme descrito no Standard Methods (APHA, 2005). Esta metodologia foi aferida em quintuplicatas analíticas utilizando uma solução padrão (Combicheck 20) com $750 \pm 75 \mathrm{mg} \mathrm{O}_{2} \mathrm{~L}^{-}$ ${ }^{1}$. O resultado médio obtido foi de $710 \pm$ $23,7 \mathrm{mg} \mathrm{O}_{2} \mathrm{~L}^{-1}$. O carbono orgânico total (COT) foi determinado utilizando um analisador de carbono orgânico total (Shimadzu, modelo TOC-VCPH). A turbidez foi determinada pelo método nefelométrico (Nephelometric Turbidity Unit, NTU) utilizando um turbidímetro (Tecnal, modelo TB1000). A cor foi determinada pelo método Platina-Cobalto conforme metodologia padrão descrita no Standard Methods (APHA, 2005). O pH foi medido utilizando um $\mathrm{pH}$ metro digital (Tecnal, modelo TEC-2). As absorvâncias em 430 nm (método Platina-Cobalto), $450 \mathrm{~nm}$ (método do metavanadato), $510 \mathrm{~nm}$ (método fenantrolina) e $600 \mathrm{~nm}$ (método colorimétrico de refluxo fechado) foram obtidas utilizando um espectrofotômetro UV-Vis (Shimadzu, modelo UV-1601 PC). Antes das análises, todas as amostras foram filtradas utilizando membranas de nylon $(0,45 \mu \mathrm{m}$ de porosidade e $25 \mathrm{~mm}$ de diâmetro), exceto para as determinações da DQO. Antes das análises envolvendo oxidação química, o excesso de $\mathrm{H}_{2} \mathrm{O}_{2}$ presente nas amostras foi removido adicionando uma alíquota $\left(0,1 \mathrm{~g} \mathrm{~L}^{-1}\right)$ da solução de catalase (2500 U fígado bovino $\mathrm{mg}^{-1}$ ) (Vilar et al., 2011a).

\section{RESULTADOS E DISCUSSÕES}

\subsection{Caracterização do efluente têxtil}

A Tabela 2 apresenta a caracterização do efluente têxtil utilizado neste estudo. O efluente apresentou uma coloração preta, oriundo da mistura de diversos corantes reativos utilizados no processo industrial, turbidez relativamente baixa (306 NTU), $\mathrm{pH}$ elevado (12,5), e carga orgânica moderada $\left(\right.$ DQO $=1636 \mathrm{mg} \mathrm{O} \mathrm{O}^{-1} \mathrm{e}$ COT $=638 \mathrm{mg} \mathrm{C} \mathrm{L}^{-1}$ ) excedendo os limites de despejos de águas residuais em corpos hídricos receptores (CONAMA 357, 2005).

Tabela 2. Caracterização do efluente têxtil não tratado.

\begin{tabular}{lcc}
\hline Parâmetro & Unidade & Valor \\
\hline $\mathrm{pH}$ & Escala Sörensen & 12,5 \\
$\mathrm{DQO}$ & $\mathrm{mg} \mathrm{O}_{2} \mathrm{~L}^{-1}$ & 1636 \\
$\mathrm{COT}$ & $\mathrm{mg} \mathrm{C} \mathrm{L}^{-1}$ & 638 \\
Cor & $\mathrm{g} \mathrm{Pt}^{-C o ~ L}$ & 5,1 \\
Turbidez & $\mathrm{NTU}^{-1}$ & 306 \\
Ferro dissolvido & $\mathrm{mg} \mathrm{Fe}^{2+} \mathrm{L}^{-1}$ & 1,3 \\
\hline
\end{tabular}

\subsection{Planejamento experimental e análise estatística}

Nesta etapa, o efluente pré-tratado via EC foi submetido ao tratamento no 
reator FFS nas condições apresentadas no delineamento experimental. As respostas obtidas para a redução da DQO são apresentadas na Tabela 3. Com o auxílio do software Statistica ${ }^{\circledR}$, obtém-se o modelo polinomial de $2^{\mathrm{a}}$ ordem para as respostas da redução da DQO $\left(R_{D Q O}^{E F / F F S}\right)$, conforme apresentado na Equação 2, cujos valores dos coeficientes da constante do modelo $\left(a_{0}\right)$, lineares $(a)$, quadráticos e das interações entre eles $(b$, $w$ e v), são apresentados na Tabela 4.

Tabela 3. Condições experimentais e os resultados em triplicata da redução da DQO (\%) do efluente têxtil tratado pelo sistema EC/FFS integrados.

\begin{tabular}{|c|c|c|c|c|c|c|}
\hline \multirow{2}{*}{ Exp. } & \multirow{2}{*}{$\begin{array}{l}{\left[\mathrm{H}_{2} \mathrm{O}_{2}\right]} \\
\left(\mathrm{g} \mathrm{L}^{-1}\right)\end{array}$} & \multirow{2}{*}{$\begin{array}{c}\mathrm{pH} \\
\text { inicial }\end{array}$} & \multirow{2}{*}{$\begin{array}{c}\text { Tempo } \\
\text { (min) }\end{array}$} & \multicolumn{3}{|c|}{ Redução da DQO (\%) } \\
\hline & & & & $\mathrm{DQO}_{1}$ & $\mathrm{DQO}_{2}$ & $\mathrm{DQO}_{3}$ \\
\hline 1 & 1 & 3 & 60 & 82,2 & 83,1 & 82,5 \\
\hline 2 & 1 & 3 & 120 & 79,9 & 78,8 & 77,9 \\
\hline 3 & 1 & 3 & 180 & 81,7 & 82,3 & 81 \\
\hline 4 & 1 & 6 & 60 & 76,7 & 77,3 & 75,8 \\
\hline 5 & 1 & 6 & 120 & 74,3 & 75,4 & 74,9 \\
\hline 6 & 1 & 6 & 180 & 74,2 & 74,1 & 75,7 \\
\hline 7 & 1 & 9 & 60 & 66,7 & 67,1 & 66 \\
\hline 8 & 1 & 9 & 120 & 65,9 & 65,7 & 65,8 \\
\hline 9 & 1 & 9 & 180 & 67,1 & 67,9 & 67,2 \\
\hline 10 & 4 & 3 & 60 & 98,1 & 98,9 & 99,2 \\
\hline 11 & 4 & 3 & 120 & 99,3 & 99,1 & 99 \\
\hline 12 & 4 & 3 & 180 & 97,9 & 98,9 & 98,6 \\
\hline 13 & 4 & 6 & 60 & 88,9 & 99,1 & 88,1 \\
\hline 14 & 4 & 6 & 120 & 87,7 & 98,9 & 87,8 \\
\hline 15 & 4 & 6 & 180 & 89 & 87,3 & 89,2 \\
\hline 16 & 4 & 9 & 60 & 82,2 & 88,1 & 81,5 \\
\hline 17 & 4 & 9 & 120 & 81,1 & 89,1 & 82,2 \\
\hline 18 & 4 & 9 & 180 & 83,1 & 81,3 & 83,5 \\
\hline 19 & 7 & 3 & 60 & 87,2 & 81,3 & 86,8 \\
\hline 20 & 7 & 3 & 120 & 88,1 & 83,3 & 87,9 \\
\hline 21 & 7 & 3 & 180 & 86,9 & 86,9 & 86,3 \\
\hline 22 & 7 & 6 & 60 & 81,1 & 88,4 & 81,9 \\
\hline 23 & 7 & 6 & 120 & 82,2 & 86,1 & 82,5 \\
\hline 24 & 7 & 6 & 180 & 83,1 & 81,5 & 84 \\
\hline 25 & 7 & 9 & 60 & 77,7 & 81,2 & 77,9 \\
\hline 26 & 7 & 9 & 120 & 76,5 & 84,1 & 77,1 \\
\hline 27 & 7 & 9 & 180 & 78,5 & 78,6 & 77,9 \\
\hline
\end{tabular}

Verifica-se pela Tabela 4, que os coeficientes das variáveis $\mathrm{H}_{2} \mathrm{O}_{2}\left(q_{1}\right)$, $\mathrm{pH}$ inicial $\left(q_{2}\right)$ e tempo de irradiação $\left(q_{3}\right)$ são linearmente e quadráticamente significantes na eficiência da reação FFS, exceto para os coeficientes dos termos quadráticos do $\mathrm{pH}\left(b_{22}=-0,10\right)$ e linear do tempo de irradiação $\left(a_{3}=0,13\right)$.

Todos os coeficientes da interação entre os termos lineares e quadráticos da concentração de $\mathrm{H}_{2} \mathrm{O}_{2}\left(q_{1}\right.$ e $\left.q_{1}^{2}\right)$ com o $\mathrm{pH}$ inicial $\left(q_{2}\right.$ e $\left.q_{2}^{2}\right)$ são significativos ( $p$ valor $<0,01)$ na eficiência do processo FFS.

$$
\begin{aligned}
& R_{D Q O}^{E F / F F S}=82+4 q_{1}-6,7 q_{2}+0,1 q_{3}+1,2 q_{1} \cdot q_{2}+0,3 q_{1} \cdot q_{3}+0,4 q_{2} \cdot q_{3}+5,7 q_{1}^{2}-0,1 q_{2}^{2}-0,4 q_{3}^{2} \\
& -0,4 q_{1} q_{2}^{2}-1,2 q_{1}^{2} q_{2}+0,4 q_{1} \cdot q_{3}^{2}+0,2 q_{1}^{2} q_{3}-0,2 q_{2} q_{3}^{2}+0,1 q_{2}^{2} \cdot q_{3}-0,7 q_{1}^{2} q_{2}^{2}+0,2 q_{1}^{2} q_{3}^{2}+0,02 q_{2}^{2} q_{3}^{2}
\end{aligned}
$$




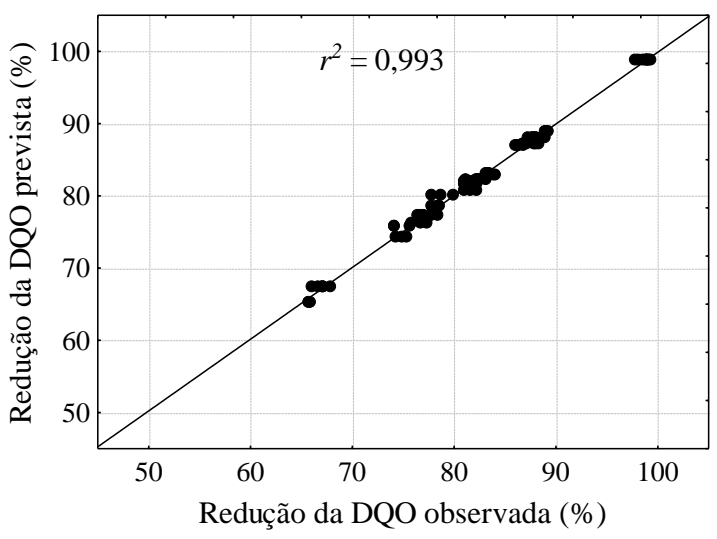

Figura 3. Correlação entre os valores observados na redução da DQO e seus valores correspondentes previstos pelo modelo estatístico proposto para o tratamento do efluente têxtil pelo sistema EF/FFS.

Os resultados apresentados pela análise estatística podem ser melhor visualizados em 3-D, fixando um dos parâmetros operacionais no valor ótimo, conforme as superfícies de respostas apresentadas na Figura 4 (a) e (b), para a eficiência do sistema integrado na redução da DQO.

As Figuras 4 (a) e (b) apresentam a elevada dependência da concentração de peróxido com tendência a obter as melhores respostas entre os níveis 3-5 g $\mathrm{H}_{2} \mathrm{O}_{2} \mathrm{~L}^{-1}$.

Com o reator FFS operando com concentrações de peróxido abaixo de $3 \mathrm{~g}$ $\mathrm{L}^{-1}$, ocorreu à diminuição da eficiência na redução da DQO pela reação foto-Fenton, provavelmente devido a pouca disponibilidade de $\mathrm{H}_{2} \mathrm{O}_{2}$ para a formação do radical hidroxila. Esta perda de eficiência também é verificada nas reações utilizando concentrações superiores a $6 \mathrm{~g} \mathrm{H}_{2} \mathrm{O}_{2} \mathrm{~L}^{-1}$.

Segundo Park et al. (2006), este efeito negativo na eficiência do processo é observado quando ocorre o aumento da concentração de $\mathrm{H}_{2} \mathrm{O}_{2}$ e pode ser explicado pela reação entre este excedente com os próprios radicais hidroxilas livres produzidos. Como resultado, o radical hidroperoxila $\left({ }^{\circ} \mathrm{O}_{2} \mathrm{H}\right)$ pode ser produzido. Estes são muito menos reativos que o radical hidroxila $\left(^{\circ} \mathrm{OH}\right)$ e não contribui para a degradação dos compostos orgânicos, conforme apresentado na Equação 3.

$$
\mathrm{H}_{2} \mathrm{O}_{2}+{ }^{\bullet} \mathrm{OH} \rightarrow{ }^{\bullet} \mathrm{O}_{2} \mathrm{H}+\mathrm{H}_{2} \mathrm{O}
$$

Além disto, podem ocorrer reações em série de consumo de radicais que eventualmente reduzem a capacidade oxidativa. O radical hidroperoxila reage com o radical hidroxila formando em sua reação moléculas de $\mathrm{H}_{2} \mathrm{O}$ e de $\mathrm{O}_{2}$, conforme apresentado na Equação 4. Não ocorrendo, desta forma, a formação do radical hidroxila, que é o principal agente na degradação dos poluentes.

$$
\cdot \mathrm{O}_{2} \mathrm{H}+\cdot \mathrm{OH} \rightarrow \mathrm{H}_{2} \mathrm{O}+\mathrm{O}_{2}
$$

A Figura 4 (a) mostra que a reação foto-Fenton é favorecida com o $\mathrm{pH}$ ácido, pois nestas condições, após a primeira oxidação dos íons ferrosos com o $\mathrm{H}_{2} \mathrm{O}_{2}$, proporciona a maior formação das espécies mais fotoativas $\mathrm{FeOH}^{2+}$ conduzindo a maior formação de espécies mais oxidantes como o radical hidroxila (Vilar et al., 2011a). Já a Figura 4 (b) apresenta que o tempo de reação FFS pouco influenciou nas respostas, pois as melhores respostas foram obtidas abaixo de $60 \mathrm{~min}$ devido à rápida cinética apresentada pelos sistemas integrados.

Segundo a interpretação da análise estatística apresentada, os melhores resultados obtidos pelo sistema EC/FFS na redução da DQO foram obtidos com 3 $\mathrm{g} \mathrm{H}_{2} \mathrm{O}_{2} \mathrm{~L}^{-1}$ e $\mathrm{pH}$ inicial 3,0. Considerando que, quando utilizado de forma isolada no tratamento do efluente têxtil, a reação FFS requer $50 \mathrm{mg} \mathrm{Fe}^{2+} \mathrm{L}^{-1}$ e $6 \mathrm{~g} \mathrm{H}_{2} \mathrm{O}_{2} \mathrm{~L}^{-1}$, a integração dos processos EC/FFS reduz o consumo de $\mathrm{H}_{2} \mathrm{O}_{2}$ e $\mathrm{Fe}^{2+}$ em 50 e $100 \%$, respectivamente.

Tabela 5. Resultados da análise de variância (2-way ANOVA) dos modelos previstos para a redução da DQO do efluente têxtil pelo sistema EC/FFS integrados, ao nível de significância de $95 \%(p<0,05)$.

\begin{tabular}{lccccccc}
\hline \multirow{2}{*}{ Parâmetro } & Fontes de & Soma dos & Graus de & Média dos & \multicolumn{2}{c}{$F$} & Nível de \\
& variação & quadrados & liberdade & quadrados & Calculado & Estatístico & Significância \\
\hline \multirow{2}{*}{ DQO } & Regressão & 5872,58 & 18 & 326,25 & 501,9 & 1,8 & $0,149 \%$ \\
& Resíduos & 40,63 & 62 & 0,65 & & & \\
& Total & 5913,21 & 80 & & & & \\
\hline
\end{tabular}

Obs.: $\mathrm{F}_{\text {cal }}>\mathrm{F}_{\mathrm{est}}=$ ajuste aceitável. 
(a)

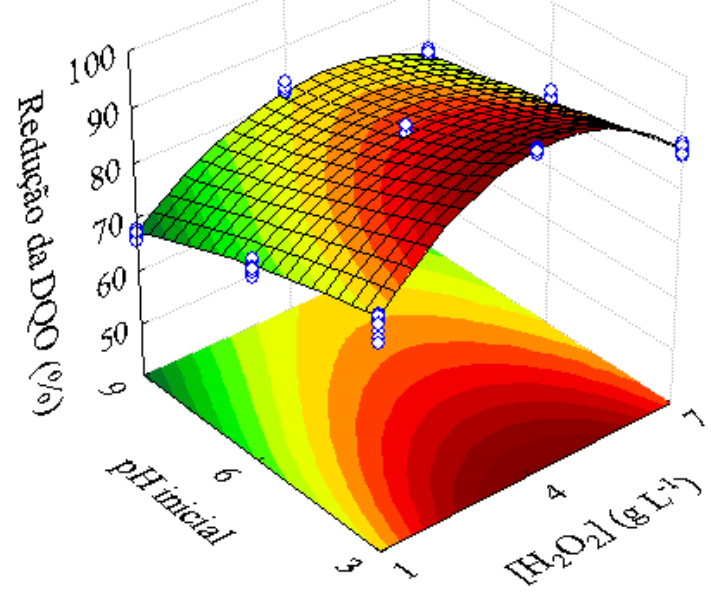

(b)

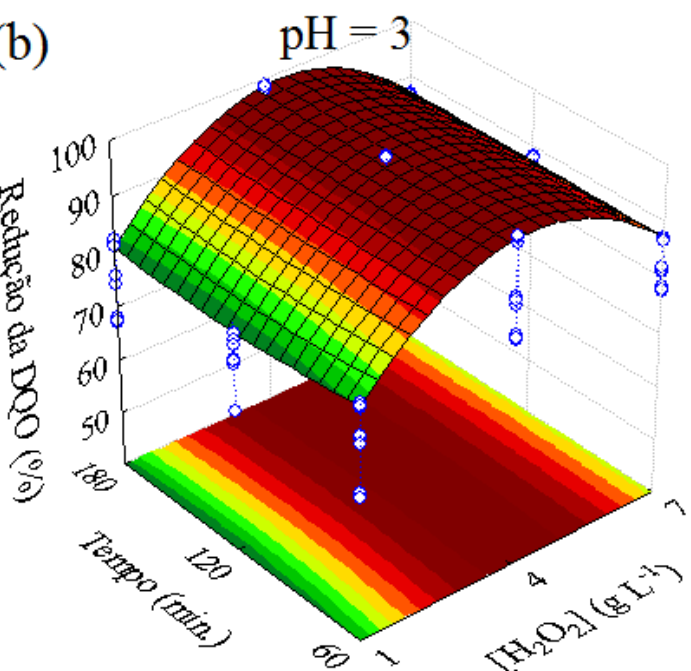

Figura 4. Superfícies de resposta dos resultados experimentais obtidos no PEC para o sistema EF/FFS no tratamento do efluente têxtil, com os dados experimentais: (a) redução da DQO em função da concentração de $\mathrm{H}_{2} \mathrm{O}_{2}\left(q_{1}\right)$, pH inicial $\left(q_{2}\right)$ e tempo de irradiação solar $\left(q_{3}\right)$ fixo em 60 min.; (b) redução da DQO em função da concentração de $\mathrm{H}_{2} \mathrm{O}_{2}\left(q_{1}\right)$, tempo de irradiação solar $\left(q_{3}\right)$ e pH inicial $\left(q_{2}\right)$ fixo em 3 .

O pré-tratamento pela EC reduziu parcialmente os compostos poluentes e a concentração inicial de peróxido requerida foi menor. Já o ferro residual da EC foi suficiente para a ocorrência da reação Fenton.

\subsection{Degradação do efluente têxtil pelos processos EC, FFS e EC/FFS}

Com os reatores operando baseado nas condições ótimas da $\mathrm{EC}(\mathrm{i}=5 \mathrm{~A} ; \mathrm{pH}$ 7), FFS (50 mg Fe ${ }^{2+} \mathrm{L}^{-1} ; 6 \mathrm{~g} \mathrm{H}_{2} \mathrm{O}_{2} \mathrm{~L}^{-1} ; \mathrm{pH}$ 3) e EC/FFS (5 min de EC; $3 \mathrm{~g} \mathrm{H}_{2} \mathrm{O}_{2} \mathrm{~L}^{-1}$; $\mathrm{pH} 3$ ) foram realizados experimentos coletando alíquotas após 5, 10, 15, 20, $30,45,60,90$ e $120 \mathrm{~min}$ para as determinações da DQO, do COT, da cor e da turbidez. Os resultados da eficiência dos processos EC, FFS e EC/FFS na redução da DQO (a), do COT (b), da cor (c) e da turbidez (d) são apresentados na Figura 5.

As Figuras 5 (a) e (b) mostram uma cinética muito mais favorável na degradação da matéria orgânica pelos processos integrados EC/FFS do que para estes de forma isolada.

A Figura 5 (a) mostra que o sistema EC/FFS integrados foi mais eficiente na redução da DQO que os processos EC e FFS, obtendo reduções de 99,5\% com 20 min. As melhores respostas obtidas pelos processos EC e FFS foram de 79 e $94 \%$ de redução da DQO com $60 \mathrm{~min}$, respectivamente.
Analisando a Figura 5 (b), percebese que as melhores respostas na redução do COT são obtidas pelo sistema EC/FFS, alcançando eficiência de 96\% em 20 min. Os processos EC e FFS apresentaram eficiência de 60 e $89 \%$ na redução do COT, respectivamente, em 120 min de reação.

Para a redução da cor e da turbidez (Figuras 5 (c) e (d), respectivamente) ambos os processos estudados mostraram elevada eficiência, com uma cinética levemente mais favorável para os processos EC/FFS integrados. Os processos EC, FFS e EC/FFS obtiveram reduções de 95,99 e $99,9 \%$ da cor com 30, 45 e 10 min, respectivamente. Já para a redução da turbidez foram alcançadas eficiências de 95, 99, e 99,9\% com 30, 60 e 10 min pelos processos EC, FFS e $\mathrm{EC} / \mathrm{FFS}$ integrados, respectivamente.

A Resolução CONAMA n ${ }^{\circ} 357$ (Brasil, 2005) estabelece os padrões de lançamento de efluentes industriais nos corpos hídricos brasileiros, fixando valores de até 100 unidades nefelométrica de turbidez, $\mathrm{pH}$ entre 6,0 e 9,0, $15 \mathrm{mg} \mathrm{Fe}$ $\mathrm{L}^{-1}$ e cor inferior a $0,075 \mathrm{mg} \mathrm{Pt}-\mathrm{Co} \mathrm{L}^{-1}$, porém não especifica os valores para os parâmetros DQO e COT. Para efluentes têxteis, sugere-se uma DQO inferior a $300 \mathrm{mg} \mathrm{O}_{2} \mathrm{~L}^{-1}$. A Tabela 6 apresenta os maiores valores de eficiência obtidos nos menores tempos de tratamento pelos processos EC, FFS e EC/FFS integrados. 

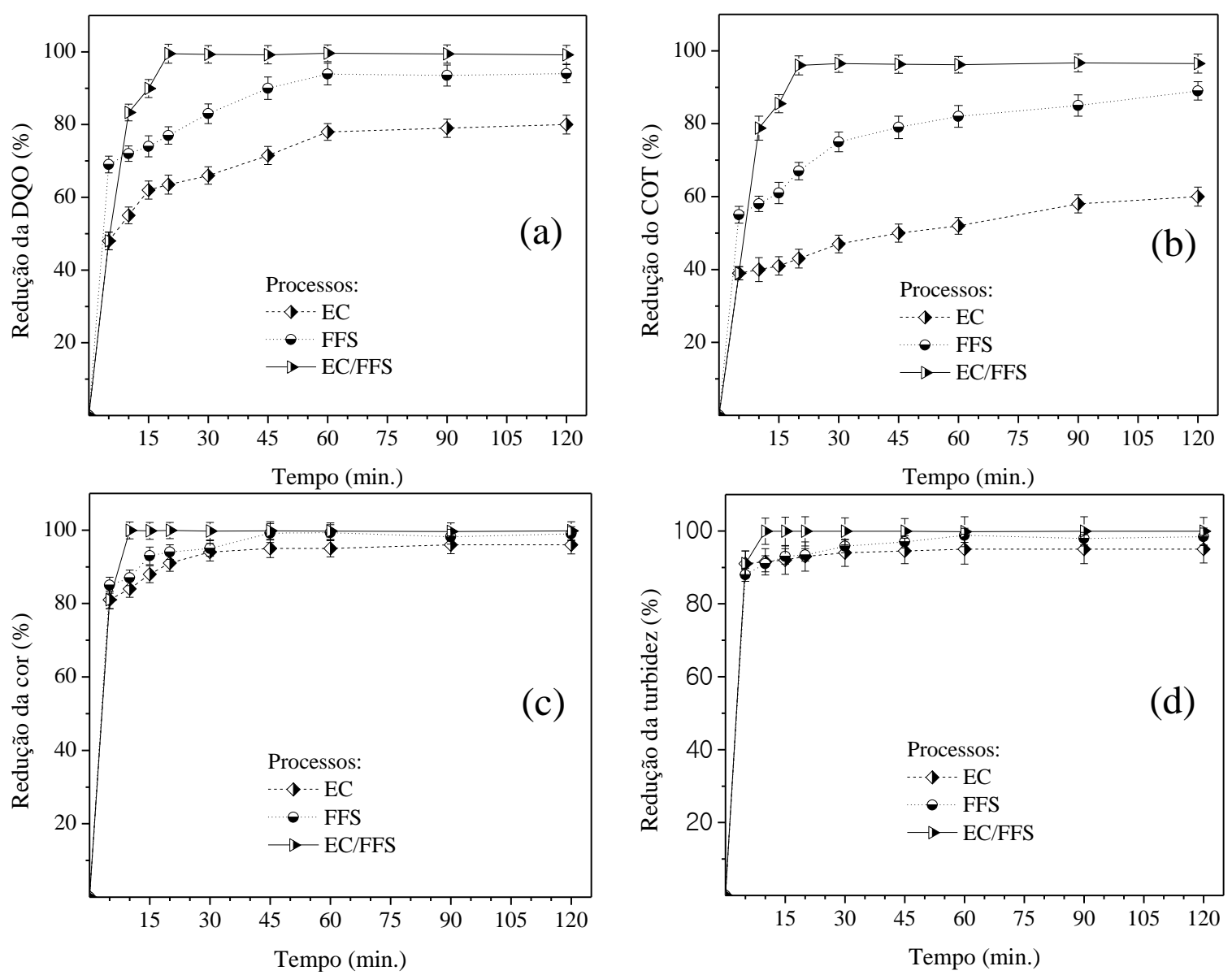

Figura 5. Perfis de redução (\%) da DQO (a), do COT (b), da cor (c) e da turbidez (d) do efluente têxtil em função do tempo reacional nos processos EC, FFS e EC/FFS integrados.

Conforme apresentado na Tabela 6, todos os processos estudados obtiveram os valores dos parâmetros avaliados inferiores aos padrões de lançamento, exceto para a EC que apresentou um efluente tratado com carga orgânica moderada $\left(\mathrm{DQO}=344 \mathrm{mg} \mathrm{O}_{2} \mathrm{~L}^{-1}\right.$ e COT $\left.=255 \mathrm{mg} \mathrm{C} \mathrm{L}^{-1}\right)$. Com isto, validou a necessidade de um processo de póstratamento a EC para maiores reduções da DQO e do COT. Sendo assim, o processo EC/FFS integrados foi à alternativa mais eficaz alcançando níveis inferiores aos padrões de lançamento de efluentes em corpos de receptores da classe III, de acordo com a Resolução CONAMA n ${ }^{\circ}$ 357/2005. Apresentando uma rápida cinética de reação, com 5 min de EC e $15 \mathrm{~min}$ de FFS, o sistema $\mathrm{EC} / \mathrm{FFS}$ obteve valores finais de $9 \mathrm{mg} \mathrm{O}_{2}$ $\mathrm{L}^{-1}$ de DQO, $26 \mathrm{mg} \mathrm{C} \mathrm{L}^{-1}$ de COT, 0,005 g Pt-Co $\mathrm{L}^{-1}$ de cor e 0,3 unidades nefelométrica de turbidez. Análises do efluente tratado pelo sistema EC/FFS apresentou concentrações de ferro superiores a $25 \mathrm{mg} \mathrm{L}^{-1}$ com o $\mathrm{pH} 3,0$. Com a correção do $\mathrm{pH}$ para > 6,0, necessário para o despejo final, a concentração de ferro reduziu para $8 \mathrm{mg}$ $\mathrm{L}^{-1}$, atendendo os padrões de lançamento impostos pela legislação. $\mathrm{O}$ restante do ferro é removido na forma de lodo, necessitando sua disposição em aterro industrial.

Tabela 6. Valores dos parâmetros físico-químicos do efluente têxtil obtidos no tempo ótimo de tratamento pelos processos EC, FFS e EC/FFS, comparados aos padrões de despejos de efluentes industriais em corpos hídricos receptores (CONAMA, 357/2005).

\begin{tabular}{lccc|cc|ccc}
\hline \multirow{2}{*}{ Parâmetro } & Efluente & \multicolumn{9}{c}{ Processo/Tempo ótimo (min) } & Padrões de \\
\cline { 3 - 7 } & bruto & EC & T.O. & FFS & T.O. & EC/FFS & T.O. & lançamento \\
\hline DQO $\left(\mathrm{mg} \mathrm{O}_{2}\right.$ L $\left.^{-1}\right)$ & 1636 & 344 & $\geq 60$ & 99 & $\geq 60$ & 9 & $\geq 5 / 15$ & n.e. \\
COT $\left(\mathrm{mg} \mathrm{C} \mathrm{L}^{-1}\right)$ & 638 & 255 & $\geq 120$ & 71 & $\geq 120$ & 26 & $\geq 5 / 15$ & n.e. \\
Cor $\left(\mathrm{g} \mathrm{Pt-Co} \mathrm{L}^{-1}\right)$ & 5,1 & 0,255 & $\geq 30$ & 0,05 & $\geq 45$ & 0,005 & $\geq 5 / 5$ & 0,075 \\
Turbidez $(\mathrm{NTU})$ & 306 & 15,3 & $\geq 30$ & 3,1 & $\geq 6$ & 0,3 & $\geq 5 / 5$ & 100 \\
\hline
\end{tabular}

n.e. : não especificado, com sugestão de valores $<300 \mathrm{mg} \mathrm{O}_{2} \mathrm{~L}^{-1}$. n.e.: não especificado. 


\section{CONCLUSÕES}

O efluente têxtil apresentou características físico-químicas acima dos limites de despejo em corpos hídricos receptores impostos pela legislação brasileira. Desta forma, após o efluente pré-tratado com $5 \mathrm{~min}$ de EC, foi realizado um planejamento experimental $3^{3}$ completo, obtendo as condições ótimas operacionais do processo FFS para o tratamento do efluente têxtil. A qualidade do modelo de $2^{\mathrm{a}}$ ordem obtido para a redução da DQO foi validada pela ANOVA e confirmada pelo bom ajuste linear dos dados experimentais.

As condições ótimas operacionais obtidas para o sistema EC/FFS integrados foram: $\mathrm{pH} 3$ e $3 \mathrm{~g} \mathrm{H}_{2} \mathrm{O}_{2} \mathrm{~L}^{-1}$. O prétratamento via $\mathrm{EC}$ possibilitou a redução no consumo do reagente $\mathrm{H}_{2} \mathrm{O}_{2}$ pelo processo FFS. Além disto, a dissolução dos eletrodos disponibilizou o ferro residual suficiente para a reação Fenton. Nestas condições, em $20 \mathrm{~min}$ de reação foram obtidas reduções de $99,5 \%$ da DQO, $96 \%$ do COT, 99,9\% da cor e da turbidez, alcançando valores inferiores aos limites de despejo em corpos hídricos receptores impostos pela legislação brasileira.

Assim, o sistema de tratamento EC/FFS integrados mostrou-se mais eficaz que os processos EC e FFS, sendo uma alternativa promissora para $\mathrm{O}$ tratamento de efluentes têxteis.

\section{AGRADECIMENTOS}

Ao $\mathrm{CNPq}$ e a CAPES (processo BEX 99794/11-5) pelo auxílio financeiro.

\section{REFERÊNCIAS}

(1988). ISO 6332:1988, Water quality Determination of iron - Spectrometric method using 1,10-phenanthroline.

AL AJI, B.; YAVUZ, Y.; KOPARAL, S. Electrocoagulation of heavy metals model wastewater using monopolar iron electrodes. Separation and Purification Technology. v. 86, p. 248-254, 2012.

APHA, AWWA, WPCF. Standard Methods for the Examination of Water and Wastewater, $21^{\text {st }}$ ed. American Public Health Association, Washington, DC. 2005 .

BABUPONNUSAMI, A.; MUTHUKUMAR, K. Advanced oxidation of phenol: A comparison between Fenton, electro-Fenton, sonoelectro-Fenton and photo-electro-Fenton processes. Chemical Engineering Journal. v. 183, p. 1-9, 2012.

BRASIL. Ministério do Meio Ambiente. Conselho Nacional do Meio Ambiente (CONAMA). Resolução no 357, de 17 de Março de 2005. Dispõe sobre a classificação e diretrizes ambientais para o enquadramento dos corpos de água superficiais, bem como estabelece as condições e padrões de lançamento de efluentes. 2005.

BORBA， F.H.; MANENTI， D.R.; MÓDENES, A.N.; MORA, N.D.; ESPINOZA-QUIÑONES, $\quad$ F.R.; PALÁCIO, S.M.; YASSUE, P.; NASCIMENTO, R. Avaliação da eficiência da técnica de eletro-floculação no tratamento de efluentes de indústrias de subprodutos avícolas. Estudos Tecnológicos. v. 6, p. 36-47, 2010.

BORBA, F.H.; MÓDENES, A.N.; ESPINOZA-QUIÑNONES, F.R.; MANENTI, D.R.; BERGAMASCO, R.; MORA, N.D. Toxicity assessment of tannery effluent treated bu an optimized photo-Fenton process. Environmental Technology, v. 34, p. 653-661, 2013.

DEVI, G.L.; RAJASHEKHAR, K.E.; RAJU, K.S.A.; KUMAR, S.G. Influence of various aromatic derivatives on the advanced photo Fenton degradation of Amaranth dye. Desalination. v. 270, p. 31-39, 2011.

ESPINOZA-QUIÑONES, $\quad$ F.R.; FORNARI, M.T.; MÓDENES, A.N.; PALÁCIO, S.M.; SILVA JR., F.G.; SZYMANSKI, N.; KROUMOV, A.D.; TRIGUEIROS, D.E.G. Pollutant removal from tannery effluent by electrocoagulation. Chemical Engineering Journal. v. 151, p. 59-65, 2009. 
ESPINOZA-QUIÑNONES,

F.R.; MÓDENES, A.N.; THEODORO, P.S.; PALÁCIO, S.M.; TRIGUEIROS, D.E.G.; BORBA, F.H.; ABUGDERAH, M.M.; KROUMOV, A.D. Optimization of the iron electro-coagulation process of $\mathrm{Cr}, \mathrm{Ni}, \mathrm{Cu}$, and $\mathrm{Zn}$ galvanization byproducts by using response surface methodology. Separation Science and Technology. v. 47, p. 688-699, 2012.

FARHADI, S.; AMINZADEH, B.; TORABIAN, A.; KHATIBIKAMAL, V.; FARD, M.A. Comparison of COD removal from pharmaceutical wastewater by electrocoagulation, photoelectrocoagulation, peroxielectrocoagulation and peroxiphotoelectrocoagulation processes. Journal of Hazardous Materials. v. 219220, p. 35-42, 2012.

FERREIRA, S.L.C.; SANTOS, W.N.L.; QUINTELLA, B.B.; NETO, B.B.; BOSQUE-SENDRA, J.M. Doehlert matrix: a chemomoetric tool for analytical chemistry - review. Talanta, v. 63, p. 1061-1067, 2004.

KATSUMATA, H.; KOIKE, $\quad$ S.; KANECO, S.; SUZUKI, T.; OHTA, K. Degradation of reactive yellow 86 with photo-Fenton process driven by solar light. Journal of Environmental Sciences. v. 22, p. 1455-1461, 2010.

KHURI, A.I.; MUKHOPADHYAY, S. Response surface methodology. Wiley Interdisciplinary Reviews: Computational Statistics, 2. p. 128-149, 2010.

LUCAS, M.S.; PERES, J.A. Decolorization of the azo dye Reactive Black 5 by Fenton and photo-Fenton oxidation. Dyes and Pigments. v. 71, p. 236-244, 2006.

MANENTI, D.R.; BORBA, F.H.; MÓDENES, A.N.; ESPINOZAQUIÑONES, F.R.; PALÁCIO, S.M.; CRESTANI, K.S.; SCHMIDT JR., P.R.; NASCIMENTO, R. Optimization of photo-Fenton process with UV irradiation in the treatment of effluents from the processing industry by-products of food. Revista de Ciências Exatas e Naturais. v. 11, p. 111-128, 2009.
MANENTI, D.R.; GOMES, L.F.S.; BORBA, F.H.; MÓDENES, A.N.; ESPINOZA-QUIÑONES, F.R.; PALÁCIO, S.M. Otimização do processo foto-Fenton utilizando irradiação artificial na degradação do efluente têxtil sintético. Engevista. v. 12, p. 22-32, 2010.

MERZOUK, B.; YAKOUBI, M.; ZONGO, I.: LECLERC, J.P.; PETERNOTTE, G.; PONTVIANNE, S.; LAPICQUE, F. Effect of modification of textile wastewater composition on electrocoagulation efficiency. Desalination. v. 275, p. 181-186, 2011.

MÓDENES, A.N.; ESPINOZAQUIÑNES, F.R.; BORBA, F.H.; MANENTI, D.R. Performance evaluation of an integrated photo-Fenton Electrocoagulatio process applied to pollutant removal from tannery effluent in batch system. Chemical Engineering Journal. v. 197, p. 1-9, 2012a.

MÓDENES, A.N.; ESPINOZAQUIÑONES, F.R.; MANENTI, D.R.; BORBA, F.H.; PALÁCIO, S.M.; COLOMBO, A. Performance evaluation of a photo-Fenton process applied to pollutant removal from textile effluent in a batch system. Journal of Environmental Management. v. 104, p. 1-8, 2012 b.

MOLLAH, M.Y.A.; SCHENNACH, R.; PARGA, J.R.; COCKE, D.L. Electrocoagulation (EC) science and applications. Journal of Hazardous Materials. v. 84, p. 29-41, 2001.

MONCAYO-LASSO, A.; RINCON, A.G.; PULGARIN, C.; BENITEZ, N. Significant decrease of THMs generated during chlorination of river water by previous photo-Fenton treatment at near neutral pH. Journal of Photochemistry and Photobiology A: Chemistry. v. 229, p. 46-52, 2012.

MONTEAGUDO, J.M.; DURÁN, A.; AGUIRRE, M.; SAN MARTIN, I. Photodegradation of Reactive Blue 4 solutions under ferrioxalate-assisted $\mathrm{UV} /$ solar photo-Fenton system with continuous addition of $\mathrm{H}_{2} \mathrm{O}_{2}$ an air 
injection. Chemical Engineering Journal. v. 162, p. 702-709, 2010.

Monteagudo, J.M.; Durán, A.; Corral, J.M.; Carnicer, A.; Frades, J.M.; Alonso, M.A. Ferrioxalate-induced solar photoFenton system for the treatment of winery wastewaters. Chemical Engineering Journal. v. 181-182, p. 281-288, 2012.

MYERS, R.H.; MONTGOMERY, D.C. Response surface methodology: process and product optimization using designed experiments, first ed. John Wiley \& Sons, New York. 2002.

NOGUEIRA, R.F.P.; OLIVEIRA, M.C.; PARTERLINI, W.C. Simple and fast spectrophotometric determination of $\mathrm{H}_{2} \mathrm{O}_{2}$ in photo-Fenton reactions using metavanadate. Talanta. v. 66, p. 86-91, 2005.

PALÁCIO, S.M.; ESPINOZAQUIÑONES, F.R.; MÓDENES, A.N.; OLIVEIRA, C.C.; BORBA, F.H.; SILVA Jr., F.G. Toxicity assessment from electro-coagulation treated-textile dye wastewaters by bioassays. Journal of Hazardous Materials. v. 172, p. 330-337, 2009.

PARK, J.H.; CHO, I.H.; CHANG, S.W. Comparasion of Fenton and PhotoFenton processes for livestock wasterwater treatment. Journal of Environmental Science and Health. v. 41, p.109-120. 2006.

PÉREZ-MOYA, M.; GRAELLS, M.; CASTELLS, G.; AMIGÓ, J.; ORTEGA, E.; BUHIGAS, G.; PÉREZ, L.M.; MANSILLA, H. Characterization of the degradation performance of the sulfamethazine antibiotic by photoFenton process. Water Research. v. 44, p. 2533-2540, 2010.

PINTOR, A.M.A.; VILAR， V.J.P.; BOAVENTURA, R.A.R. Decontamination of cork wastewaters by solar-photo-Fenton process using cork bleaching wastewater as $\mathrm{H}_{2} \mathrm{O}_{2}$ source. Solar Energy, v. 85, p. 579-587, 2011.

PRATO-GARCIA, D.; BUITRÓN, G. Evaluation of three reagent dosing strategies in a photo-Fenton process for the decolorization of azo dye mixtures. Journal of Hazardous Materials. v. 217218, p. 293-300, 2012.

ROCHA, E.M.R.; VILAR, V.J.P.; FONSECA, A.; SARAIVA, I.; BOAVENTURA, R.A.R. Landfill leachate treatment by solar-driven AOPs. Chemical Engineering Journal. v. 192, p. 1-9, 2012.

ROSALES, E.; PAZOS, M.; SANROMÁN, S.A. Advances in the electro-Fenton process for remediation of recalcitrant organic compounds. Chemical Engineering Technology. v. 35, p. 609-617, 2012.

VILAR， V.J.P.; CAPELO, S.M.S.; SILVA, T.F.C.V.; BOAVENTURA, R.A.R. Solar photo-Fenton as a preoxidation step for biological treatment of landfill leachate in a pilot plant with CPCs. Catalysis Today. v. 161, p. 228234, 2011a.

VILAR, V.J.P.; PINHO, L.X.; PINTOR, A.M.A.; BOAVENTURA , R.A.R. Treatment of textile wastewaters by solar-driven advanced oxidation process. Solar Energy. v. 84, p. 1927-1934, 2011b.

WANG, Z.; KUTAL, C. Photocatalytic mineralization of 2,4,6-trinitrotoluene in aqueous suspensions of titanium dioxide. Chemosphere. v. 30, p. 1125-1136, 2001.

YEBER, M. C.; FREER, J.; BAEZA, J.; MANSILLA, H.D. $\mathrm{TiO}_{2}$ and $\mathrm{ZnO}$ thin film formation on glass. Advanced Wastewater Treatment, Recycling and Reuse. v. 2, p. 907-911, 1998.

YILMAZ, A.E.; BONCUKCUOGLU, R.; KOCAKERIM, M.; KARAKAS, I.H. Waste utilization: the removal of textile dye (Bomaplex Red CR-L) from aqueous solution on sludge waste from electrocoagulation as adsorbent. Desalination. v. 277, p. 156-163, 2011. 